\title{
DEVELOPMENT OF ONE METER-LONG LITHIUM PLASMA SOURCE AND EXCIMER MODE REDUCTION FOR PLASMA WAKEFIELD APPLICATIONS
}

\author{
S. DiMaggio, L. Archambault, P. Catravas, P. Volfbeyn, and W. P. Leemans, ${ }^{\vee}$ LBNL, Berkeley, CA 94720 \\ K. Marsh, P. Muggli, S. Wang, and C. Joshi, UCLA, Los Angeles, CA 90024
}

\begin{abstract}
A one meter long plasma source has been developed for studies at the Stanford Linear Accelerator Center (SLAC) of plasma wakefield excitation by a $30 \mathrm{GeV}$ electron beam in an extended plasma column. The plasma is formed by ionization of a Li-vapor with an ArF EXCIMER laser $(193 \mathrm{~nm})$. The Li-vapor is produced in a heat pipe oven which will be installed in the electron beam transport line. Through control of the oven temperature, neutral vapor densities reaching $2 £ 10^{15} \mathrm{~cm}^{i^{3}}$ are produced. We report the details of the oven construction and temperature profile measurements with and without Lithium vapor. In the experiment, the EXCIMER laser will be located about $15 \mathrm{~m}$ away from the plasma source. Beams produced by EXCIMER lasers operating with a stable resonator cavity are unsuitable due to the large number of modes present in such beams. A significant reduction of the modal content has been obtained through the use of an unstable resonator design. Results of the implementation of this cavity configuration on the propagation characteristics of the EXCIMER beam will be presented, including the design of the final telescope for spot size reduction.
\end{abstract}

\section{INTRODUCTION}

A one-meter long plasma source has been constructed which will permit the properties of extended plasma columns as optical elements in beamlines to be studied in an operating regime at $30 \mathrm{GeV}$. The E157 Experiment[1, 2, 3], to take place at the Final Focus Test Beam (FFTB) at the Stanford Linear Accelerator, utilizes a single $30 \mathrm{GeV}$ electron bunch with a bunchlength of $z \ldots 400 ; 600, \mathrm{~m}$ and up to $4 \times 10^{10}$ electrons, which will travel through a full meter of plasma. Wakefields excited by the head of the bunch are expected to provide an accelerating gradient to the bunch tail. For efficient wakefield excitation, frequency content at $!_{p}$ is provided by matching the beam bunchlength to the plasma period, $\dot{c}_{b} \gg 1=!_{p}$. The wake amplitude expected for the optimal plasma density range of $2 \times 10^{14} \mathrm{~cm}^{i^{3}}$ is $0.5 \mathrm{GeV} / \mathrm{m}$. Calculations show that the plasma ions will exert focusing forces on the electron beam capable of dramatically changing the bunch transverse size and divergence[2]. Experimental studies of these mechanisms have been performed at lower beam energies (10's

Work supported by the U.S. Department of Energy under contract No. DE-AC-03-76SF0098.

y Email:wpleemans@1bl.gov of $\mathrm{MeV}$ ) and wakefield acceleration of the bunch tail has been demonstrated [4]. The two key components for the experiment are the 1 meter plasma source and the beam diagnostics. Here, the practical implementation of the full length source for the E157 experiment is described.

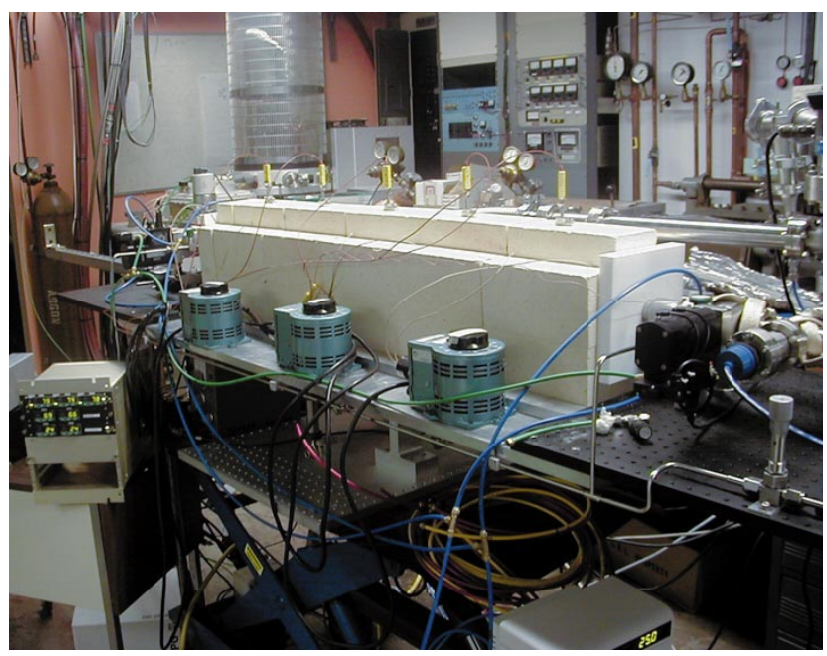

Figure 1: One meter long Lithium heat pipe oven.

The minimum plasma requirements are the following: length of at least 1 meter, density of $2-5 \times 10^{14} \mathrm{~cm}^{i^{3}}$ and a transverse dimension on the order of a few $\mathrm{mm}$, large compared with the electron beam spot size of 》50 microns at the plasma entrance. To provide a plasma of the appropriate density and dimensions, while avoiding beaminduced ionization, Lithium was chosen. The ionization potential of Lithium $(5.39 \mathrm{eV})$ is less than the photon energy at $193 \mathrm{~nm}(5.4 \mathrm{eV})$ and it can therefore be singly ionized using an $\mathrm{ArF}$ based Excimer laser. Typical fluences required are $30-50 \mathrm{~mJ} / \mathrm{cm}^{2}$ to produce a fractional ionization of the neutral vapor by the laser on the order of 10$20 \%$. Beam induced ionization (impact of $30 \mathrm{GeV}$ electrons as well as background electrons) is expected to be less than a percent and its effect should therefore be minimal. In order to obtain a Lithium plasma with the appropriate density, the Lithium vapor temperature must be raised to $750^{-} \mathrm{C}$. Accordingly, the plasma source is constructed in heat pipe oven configuration[5, 6, 7]. The 1 meter oven built at LBNL and described below is based on the design of a 0.25 meter prototype built at UCLA.[8] 


\section{OVEN DESCRIPTION AND CHARACTERIZATION}

The heat pipe oven provides a homogeneous metal vapor over a well defined length. The vapor evaporates from melted metal uniformly distributed by capillary action along a wick lining the pipe interior. Metal vapor pressure is defined by that of an inert gas displaced from the heated length of pipe to cold pipe ends, where it forms a gaseous plug which can be easily monitored[5, 6, 7].

The 1 meter long heat pipe oven shown in Figure 1 was constructed of a 56 " long stainless steel tube 1.5 inches in diameter with an inside lining of $31 / 2$ turns of fine stainless steel mesh. The pipe exterior is insulated with double layer of oven brick. A total of three independently powered heater tapes are wrapped around the exterior of the oven pipe. The temperature profile is provided by an exterior array of five K-type (Chromel-Alumel) thermocouples evenly spaced along the 1 meter length with spring loading to improve contact with the heat pipe surface. Automatic temperature regulation is performed by monitoring the readouts of three of the exterior thermocouples and adjusting the heater tapes to maintain any input setpoint up to $750^{-} \mathrm{C}$. Heater tape power is interlocked to the temperature of the water jackets on both ends of the oven.

The temperature profile measured in the oven without Lithium is shown in Figure 2 and was measured by drawing a $\mathrm{K}$ type thermocouple braced inside a $3 / 8$ inch stainless steel pipe through the oven. The corresponding exterior thermocouple array profile is shown in Figure 3a, normalized to the oven central temperature. Both measurements provide evidence that the temperature profile taper extends at least $25 \mathrm{~cm}$ from the water jackets (circulating at $30^{-} \mathrm{C}$ ) into the oven interior when the oven does not contain Lithium.

Lithium (40 g) was then added to the oven and sealed unit was filled with Helium. Raising the oven temperature results in the change in temperature profile shown in Figure $3 b$. The reduction in boundary effects and flattening of the profile indicates that Lithium vapor extends along a one meter length at the desired density, corresponding to a $\mathrm{He}$ pressure of $200 \mathrm{mTorr}$.

\section{EXCIMER MODE CONTENT REDUCTION}

While readily available Excimer lasers operating with ArF do provide the necessary photon energy to ionize the plasma, in practice, the use of an Excimer introduces a significant complication. To form a meter-long plasma, the Excimer spot size must be maintained constant over a distance of 1-2 meters, a requirement in conflict with the high mode content typical of Excimers. The original Excimer cavity configuration produced the tophat intensity profile and could not have been coupled into the beamline without significant loss, as the beam diameter, .... w, two meters from a waist of $5 \mathrm{~mm}$ was found to be 4 inches. In ad-

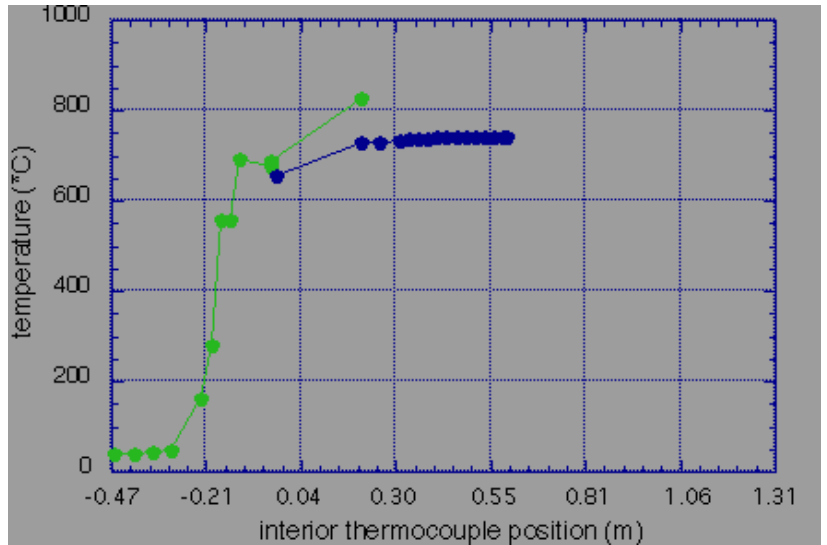

Figure 2: Oven temperature profiles in vacuum (i.e. without Lithium) measured with interior thermocouple for two temperature setpoints reaching or exceeding the desired operating temperature of $750^{-} \mathrm{C}$.

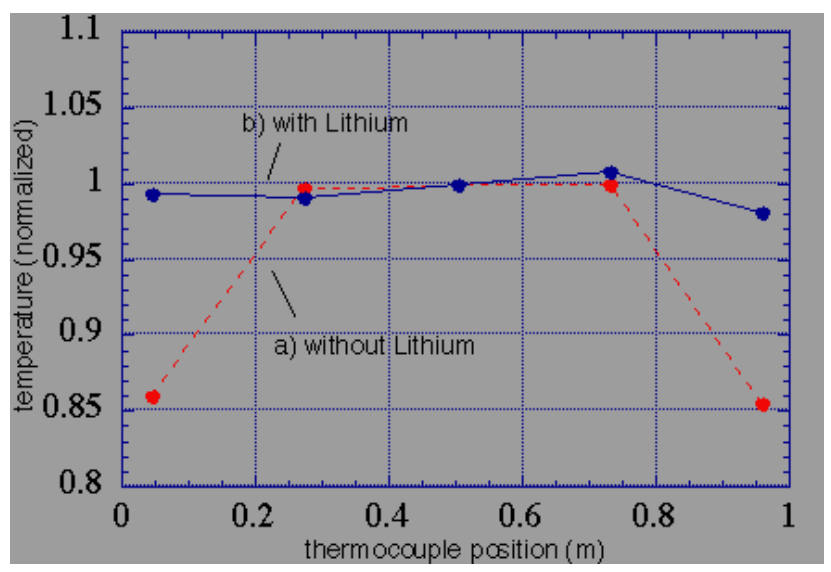

Figure 3: Exterior thermocouple array readouts (normalized to central oven temperature) with and without Lithium as a function of distance along the oven. Note the reduction in edge effects, a signature of the presence of Lithium vapor.

dition, the high modal content can lead to loss of power in long transport lines if the higher order modes diffract out of the system. For the E-157 Experiment at SLAC, the physical layout of the beamline and surrounding structures requires that the laser and plasma source be separated by a minimum of 15 meters.

We addressed this problem by converting the Excimer cavity from a stable to an unstable resonator design to reduce mode content. The unstable cavity resonator consists of a planar Aluminum coated high reflector and an uncoated meniscus lens as the outcoupler, followed by a collimating lens. The measured mode propagation was consistent with a waist located at the virtual focus of the meniscus lens, as expected from simple ray tracing.

To simulate transport losses for the unstable resonator configuration, power loss as a function of distance was measured using only a single collimating lens positioned 


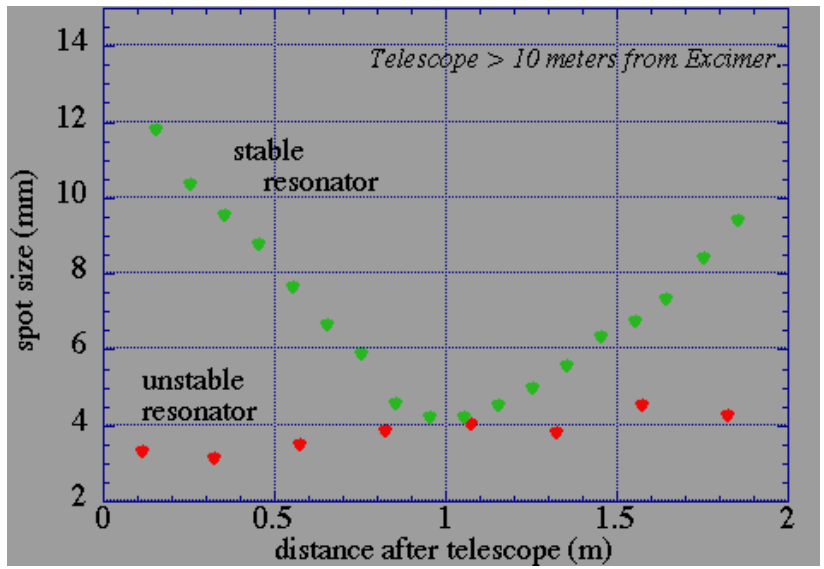

Figure 4: Comparison of mode propagation for $5 \mathrm{~mm}$ spot size at $248 \mathrm{~nm}$ for stable and unstable resonators shows significant reduction in mode content.

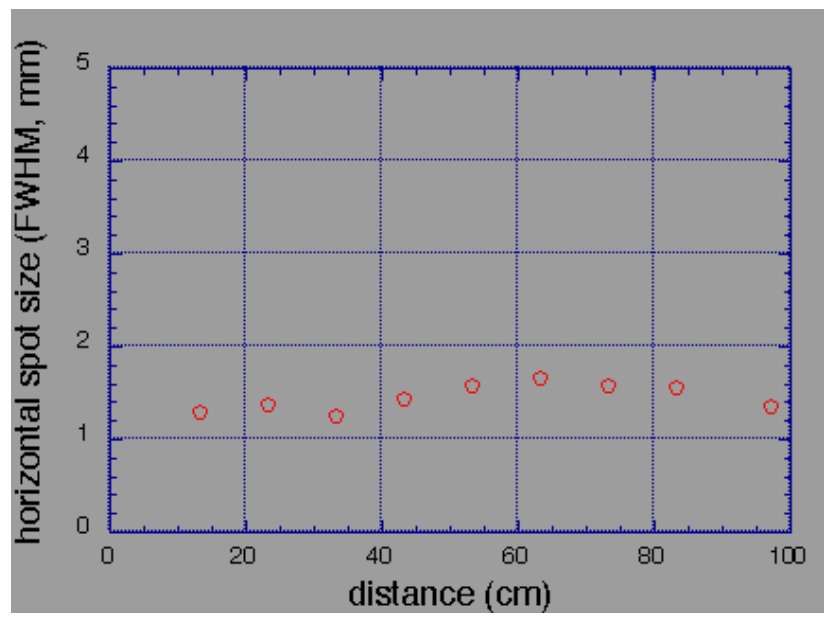

Figure 5: Test of demagnifying telescope for final focus of Excimer at $193 \mathrm{~nm}$ into oven: evolution of Excimer mode at $193 \mathrm{~nm}$ shows only minor change in spot size over one meter propagation.

about $10 \mathrm{~cm}$ from the Excimer output port. $120 \mathrm{~mJ}$ at $248 \mathrm{~nm}$ propagated for 18 meters without significant loss of power into the 2" aperture, after an initial drop by about a factor of 2 in the first two meters. The initial drop may be reduced through the use of 3" optics.

The mode content figures of merit were quantified by performing systematic scans of the beam profile through a waist. The intensity profile itself was measured by imaging the fluorescence produced by the $193 \mathrm{~nm}$ radiation in a BK7 substrate onto a CCD camera. A beamsplitter placed in the Excimer path prevented damage to the substrate from the small spot sizes. The profile corresponding to the stable resonator, which had a mode content, $\mathrm{M}^{2}$ of 990 at 248 $\mathrm{nm}$; the $\mathrm{M}^{2}$ figure for the unstable resonator was improved to less than fifty (Figure 4). Mode propagation at $193 \mathrm{~nm}$ over a one meter length after demagnification to a spot size between 1 and $2 \mathrm{~mm}$ is shown in Figure 5, confirming that the reduced mode content permits the small spot size to be maintained over the full length required for the plasma source.

The final coupling of the Excimer into the beamline will be implemented using thin (150-300 micron) fused silica foils with a high reflecting $193 \mathrm{~nm}$ dielectric coating on one side to be located 1-2 meters from the oven center. For the planned wakefield experiments, the Excimer incoupling and outcoupling foils sit directly in the path of the $30 \mathrm{GeV}$ electron beam, which must traverse not only these two foils but two Beryllium foils. The Be foils form the barrier between the heat pipe oven, which operates at a pressure of 200 mTorr, and the ultra-high vacuum of the surrounding beampipe. The potential for scattering and for damage to the foils has been considered. Scattering from a 300 micron fused-silica substrate is expected to be small, increasing an initial beam divergence from 2.5-10 "rad to a little over 20 "rad. The foils have been tested in the beamline without visible signs of damage. As an additional note, the foils are back-coated with an Aluminum substrate and serve a double role as an OTR beam diagnostic as well as Excimer mirrors, taking advantage of their proximity to the oven entrance and exit [3].

In summary, we have developed one-meter long plasma source suitable for experiments to investigate the plasma wakefield excitation of an extended plasma column by a $30 \mathrm{GeV}$ electron beam. The source is designed to provide a plasma density of $10^{14} \mathrm{~cm}^{i}{ }^{3}$ over dimensions of a few $\mathrm{mm}{ }^{2}$ by 1 meter. Temperature profiles have been recorded, and the signature of appropriate densities of Lithium vapor has been observed in the temperature profile boundary. In order to provide a Lithium plasma of the required dimensions, the Excimer cavity resonator was reconfigured to solve the problems caused by high mode content. Measurements testing the performance of a demagnifying telescope confirmed that that the Excimer spot could be maintained at 1-2 mm width over extended distances.

\section{ACKNOWLEDGMENTS}

The authors acknowledge useful conversations with $\mathrm{K}$. Kennedy and the assistance from J. Ayers during the development of the oven and suggestions from Mike Skaggs of Lambda Physik for modification of the Excimer cavity.

\section{REFERENCES}

[1] R. Assman et al., these Proceedings, WEBL6.

[2] R.G.Hemker et al., these Proceedings, FRA124

[3] P. Catravas et al., these Proceedings, WEA100.

[4] N. Barov et al., Phys. Rev. Lett., 80, 81-4 (1998); Ibid., these Proceedings, WEBL5.

[5] C.R. Vidal and J. Cooper, J. Appl. Phys., 40, 3370-3374 (1969).

[6] G. M. Grover et al., J. Appl. Phys. 35, 1990 (1964)

[7] M. Bacal and W. Reichelt, Rev. Sci. Instrum. 45, 769 (1974).

[8] P. Muggli, these Proceedings, FRA115. 